\title{
Dermatofibrosarcoma protuberans: A clinical analysis
}

\author{
ANQI LYU and QIYING WANG
}

Department of Plastic Surgery, The First Affiliated Hospital of Zhengzhou University, Zhengzhou, Henan 450052, P.R. China

Received June 21, 2017; Accepted April 24, 2018

DOI: $10.3892 / \mathrm{ol} .2018 .8802$

\begin{abstract}
Dermatofibrosarcoma protuberans (DFSP) is a rare cutaneous tumor with a high incidence of misdiagnosis. DFSP has a high rate of recurrence but a low rate of metastasis. In the present study, retrospective analyses were performed on the clinical features, differential diagnosis and treatment of patients with DFSP to improve our understanding of the disease and allow more effective treatment measures to be implemented. The present study investigated the clinicopathological features of 70 pathologically confirmed cases of DFSP at the First Affiliated Hospital of Zhengzhou University (Zhengzhou, China) between March 2012 and 2017. The primary endpoint was recurrence rate. Three cases were analyzed in detail. The results revealed that 7 of the 41 primary patients (follow-up at 2.7 years) had recurrence (17.1\%), compared with 11 (37.9\%) of the 29 recurrent patients (follow-up at 2.0 years, $\mathrm{P}=0.049$ ). Of the 59 patients with DFSP (follow-up at 2.6 years), 12 had recurrence $(20.3 \%)$ compared with $6(54.6 \%)$ of the 11 patients with fibrosarcomatous DFSP (FS-DFSP; follow-up at 2.1 years, $\mathrm{P}=0.045)$. DFSP requires diagnosis by pathological examination, and surgical resection is the main treatment. DFSP demonstrated a high recurrence rate, with the degree of malignancy increasing following multiple recurrences. FS-DFSP had a higher risk of local recurrence and distant metastasis, and a higher degree of malignancy than classic DFSP. These data may be useful to guide clinicians to improve decisions in the treatment of patients with DFSP.
\end{abstract}

\section{Introduction}

Dermatofibrosarcoma protuberans (DFSP) is a low-grade soft tissue tumor occurring in the dermis and subcutaneous tissues,

Correspondence to: Dr Qiying Wang, Department of Plastic Surgery, The First Affiliated Hospital of Zhengzhou University, 50 Jianshe East Road, Zhengzhou, Henan 450052, P.R. China E-mail: wangqiying@zzu.edu.cn

Abbreviations: DFSP, dermatofibrosarcoma protuberans; FS-DFSP, fibrosarcomatous DFSP; MMS, Mohs micrographic surgery

Key words: dermatofibrosarcoma protuberans, fibrosarcomatous dermatofibrosarcoma protuberans, recurrence which accounts for $\sim 1 \%$ of all soft tissue sarcomas $(1,2)$. It was initially characterized as a keloid-like sarcoma (3), although Hoffman gave its current name in 1925 (4).

DFSP usually occurs in young to middle-aged patients but can present in all age groups (5). It is commonly found on the trunk, however, it can also develop in the extremities, head or neck. DFSP demonstrates local infiltrative growth but seldom metastasizes distally (6). DFSP is divided histopathologically into classical and non-classical types (7). Classical-type DFSP typically forms a radial or storiform pattern, with the cancer tissue extending into the subcutaneous fat and forming a honeycomb-like structure (8). Atypical DFSP comprises at least 10 subtypes, of which the most common include pigment type, mucus type, and sarcoma type (6). Fibrosarcomatous DFSP (FS-DFSP) is also an atypical DFSP subtype, with high rates of recurrence and metastasis.

The early clinical symptoms of DFSP are non-specific, making diagnosis difficult and leading to a high incidence of misdiagnosis. Pathological and immunohistochemical examinations are thus currently the gold standard for diagnosing DFSP, with surgical resection remaining the main treatment option. In the present study, 70 cases of DFSP were retrospectively analyzed and their clinical features, differential diagnosis, and treatment were investigated.

\section{Patients and methods}

Patient information. The study group comprised 70 patients, including 41 primary cases at first diagnosis and 29 recurrent cases. There were equal numbers of male and female patients. Patient ages ranged between 5 and 76 years (mean age, 43 years). The tumor was located on the trunk in 40 patients, the extremities in 19 patients, and the head and neck in 11 patients. The disease course duration ranged between 1 month and 40 years. The maximal tumor diameter ranged between 0.5 and $15 \mathrm{~cm}$.

Clinical manifestations and diagnosis. The main clinical manifestations were pale red or brown irregular indurations, showing slow growth. They appeared either as solitary nodules or as multiple scattered confluent masses. The skin lesions lacked typical characteristics. In total, 59 cases were diagnosed as DFSP and 11 cases were diagnosed as FS-DFSP.

Therapy. All 70 patients underwent surgical treatment. In cases with no preoperative pathological diagnosis, the tumors underwent rapid intraoperative freezing to determine 
Table I. Baseline characteristics and recurrence rates of primary and recurrent cases.

\begin{tabular}{|c|c|c|c|c|c|}
\hline \multirow[b]{2}{*}{ Characteristic } & \multirow[b]{2}{*}{$\mathrm{n}$} & \multicolumn{2}{|c|}{ Primary cases } & \multicolumn{2}{|c|}{ Recurrent cases } \\
\hline & & $\mathrm{n}$ & $\%$ & $\mathrm{n}$ & $\%$ \\
\hline Cases & 70 & 41 & 58.6 & 29 & 41.4 \\
\hline \multicolumn{6}{|l|}{ Sex } \\
\hline Male & 35 & 20 & 48.8 & 15 & 51.7 \\
\hline Female & 35 & 21 & 51.2 & 14 & 48.3 \\
\hline \multicolumn{6}{|l|}{ Site } \\
\hline Head and neck & 11 & 6 & 14.7 & 5 & 17.2 \\
\hline Trunk & 40 & 24 & 58.5 & 16 & 55.2 \\
\hline Extremity & 19 & 11 & 26.8 & 8 & 27.6 \\
\hline \multicolumn{6}{|l|}{ Age (years) } \\
\hline$<40$ & 18 & 11 & 26.8 & 7 & 24.1 \\
\hline $40-50$ & 40 & 23 & 56.1 & 17 & 58.6 \\
\hline$>50$ & 12 & 7 & 17.1 & 5 & 17.3 \\
\hline \multicolumn{6}{|l|}{ Tumor size $(\mathrm{cm})^{\mathrm{a}}$} \\
\hline$<1$ & 19 & 14 & 34.1 & 5 & 17.3 \\
\hline $1-2$ & 33 & 20 & 48.8 & 13 & 44.8 \\
\hline $2+$ & 18 & 7 & 17.1 & 11 & 37.9 \\
\hline \multicolumn{6}{|l|}{ Recurrence } \\
\hline Yes & 18 & 7 & 17.1 & 11 & 37.9 \\
\hline No & 52 & 34 & 82.9 & 18 & 62.1 \\
\hline
\end{tabular}

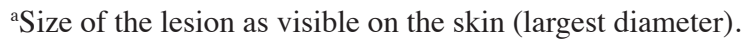

the nature of the tumor. If the tumor was determined to be a low-grade malignant spindle cell tumor, resection was extended to $3 \mathrm{~cm}$, and intraoperative frozen sections were obtained from all directions to confirm that the margins were negative. A free skin graft or partial flap was used to repair the wound following tumor resection. In addition, five patients were administered with a continuous course of radiotherapy if required, at a dose of 50.0-60.0 Gy.

Hematoxylin and eosin staining. Samples were fixed in $40 \%$ formaldehyde at $25^{\circ} \mathrm{C}$ for $12 \mathrm{~h}$. The reagents used were hematoxylin, eosin, $0.5 \%$ hydrochloric acid alcohol solution and $0.2 \%$ ammonia solution ( $\mathrm{pH}$ between $7.5-8$ ). The sections were sliced $3 \mu \mathrm{m}$ thick and heated in a microwave oven for $1 \mathrm{~h}$ at $60^{\circ} \mathrm{C}$. The dewaxing was performed by adding: Dimethylbenzene for $10 \mathrm{~min}$, three times; anhydrous ethanol for $5 \mathrm{~min}$, two times; $95 \%$ ethanol for $5 \mathrm{~min}$; $90 \%$ ethanol for $5 \mathrm{~min}$; $80 \%$ ethanol for $5 \mathrm{~min}$; $75 \%$ ethanol for $5 \mathrm{~min}$; and distilled water for $5 \mathrm{~min}$. Staining was performed by adding: i) Hematoxylin for $10 \mathrm{~min}$; ii) distilled water, for $1 \mathrm{~min}$; iii) $0.5 \%$ hydrochloric acid alcohol solution for $20 \mathrm{sec}$; iv) distilled water for $2 \mathrm{~min}$; v) $0.2 \%$ ammonia for $40 \mathrm{sec}$; vi) distilled water for $2 \mathrm{~min}$; vii) $0.5 \%$ eosin for $5 \mathrm{~min}$; and viii) distilled water for $30 \mathrm{~min}$. Dehydration was performed by adding $80 \%$ ethanol for $3 \mathrm{~min}, 90 \%$ ethanol for $3 \mathrm{~min}$, 95\% ethanol for $3 \mathrm{~min}$, anhydrous ethanol for $5 \mathrm{~min}$ and fresh anhydrous ethanol for $5 \mathrm{~min}$. Fresh xylene was added for $5 \mathrm{~min}, 3$ times, to remove ethanol. Samples were sealed with neutral gum. The sealed slices were placed incubated at
Table II. Baseline characteristics and recurrence rates of different pathological types.

\begin{tabular}{|c|c|c|c|c|c|}
\hline \multirow[b]{2}{*}{ Characteristic } & \multirow[b]{2}{*}{$\mathrm{n}$} & \multicolumn{2}{|c|}{ DFSP } & \multicolumn{2}{|c|}{ FS-DFSP } \\
\hline & & $\mathrm{n}$ & $\%$ & $\mathrm{n}$ & $\%$ \\
\hline Patients & 70 & 59 & 84.3 & 11 & 15.7 \\
\hline \multicolumn{6}{|l|}{ Sex } \\
\hline Male & 35 & 30 & 50.8 & 5 & 45.4 \\
\hline Female & 35 & 29 & 49.2 & 6 & 54.6 \\
\hline \multicolumn{6}{|l|}{ Site } \\
\hline Head and neck & 11 & 8 & 13.6 & 3 & 27.2 \\
\hline Trunk & 40 & 34 & 57.6 & 6 & 54.6 \\
\hline Extremity & 19 & 17 & 28.8 & 2 & 18.2 \\
\hline \multicolumn{6}{|l|}{ Age (years) } \\
\hline$<40$ & 18 & 16 & 27.1 & 2 & 18.2 \\
\hline $40-50$ & 40 & 33 & 55.9 & 7 & 63.6 \\
\hline$>50$ & 12 & 10 & 17.0 & 2 & 18.2 \\
\hline \multicolumn{6}{|l|}{ Tumor size $(\mathrm{cm})^{\mathrm{a}}$} \\
\hline$<1$ & 19 & 15 & 25.4 & 4 & 36.4 \\
\hline $1-2$ & 33 & 29 & 49.2 & 4 & 36.4 \\
\hline $2+$ & 18 & 15 & 25.4 & 3 & 27.2 \\
\hline \multicolumn{6}{|l|}{ Recurrence } \\
\hline Yes & 18 & 12 & 20.3 & 6 & 54.6 \\
\hline No & 52 & 47 & 79.7 & 5 & 45.4 \\
\hline
\end{tabular}

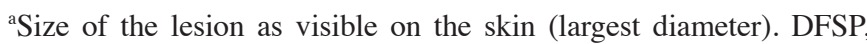
dermatofibrosarcoma protuberans; FS-DFSP, fibrosarcomatous DFSP. 
Table III. Comparison of recurrence rates in different pathological types.

\begin{tabular}{lccc}
\hline Type & Recurrence, $\mathrm{n}(\%)$ & No recurrence, $\mathrm{n}(\%)$ & $\chi^{2}$-value \\
\hline DFSP & $12(20.33)$ & $47(79.67)$ & 4.030 \\
FS-DFSP & $6(54.55)$ & $5(45.45)$ & 0.045
\end{tabular}

DFSP, dermatofibrosarcoma protuberans; FS-DFSP, fibrosarcomatous DFSP.

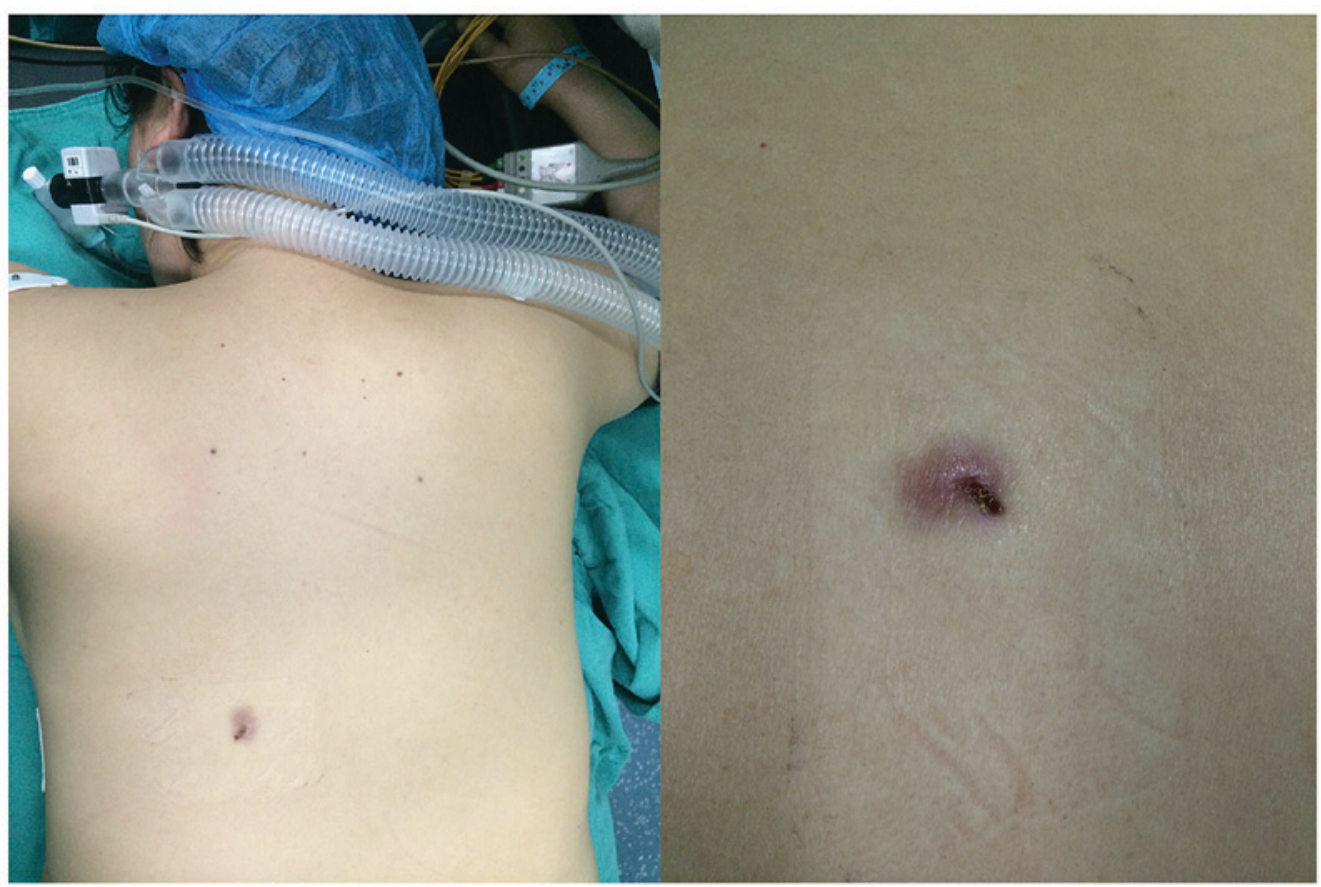

Figure 1. Patient with an atypical skin lesion.

$37^{\circ} \mathrm{C}$. Following drying, the sections were observed under a light microscope (magnifications, $\mathrm{x} 40, \mathrm{x} 100, \mathrm{x} 200$ and $\mathrm{x} 400$ ). Unless specified, all step were performed at room temperature.

Statistical analysis. Statistical analysis was performed using IBM SPSS Statistics 21.0 software (IBM SPSS, Armonk, NY, USA). Descriptive statistics were used to report patients' baseline characteristics. The clinical features and outcomes of cases treated by pathological type were compared using the $\chi^{2}$ test. All tests were two-sided, with $\alpha=0.05$.

\section{Results}

Among the 70 cases, 41 were first diagnosed in The First Affiliated Hospital of Zhengzhou University (Zhengzhou, China), and 29 were referred to The First Affiliated Hospital of Zhengzhou University for the treatment of recurrence, following treatment in other hospitals. The skin flap or skin graft survived well in all cases, with only one case of partial necrosis. The follow-up period ranged between 3 and 36 months, with 18 cases of recurrence (Tables I and II), and three cases of distant metastasis, one of which was DFSP and two were FS-DFSP, with one of the latter succumbing to mortality. Of the 41 primary patients (follow-up at 2.7 years), seven had recurrences (17.1\%), compared with 11 (37.9\%) of the 29 recurrent patients (follow-up at 2.0 years, $\mathrm{P}=0.049$ ). The recurrence rate was significantly higher among the referred cases compared with the newly diagnosed cases. In terms of the pathological types, 12 of the 59 DFSP patients (follow-up 2.6 years) had recurrences $(20.3 \%)$, compared with six $(54.6 \%)$ of the 11 patients with FS-DFSP (follow-up 2.1 years, $\mathrm{P}=0.045$ ) (Table III). The recurrence rate was significantly higher among patients with FS-DFSP compared with those with DFSP. The cases reported here include one patient with an atypical skin lesion (Fig. 1), who received a definitive diagnosis of DFSP following a biopsy at his first visit, and who subsequently underwent extensive resection with no recurrence in the following 3 years.

The follow three cases are representative: Case 1, DFSP with multiple recurrences and metastasis; case 2, FS-DFSP; case 3 , classic DFSP, with pathogenesis being representative.

Case 1. Case 1 was a 42-year-old woman with a history of subtotal thyroidectomy for a thyroid tumor (unknown nature) in 2006 , which left a $0.5 \times 0.3 \mathrm{~cm}$ mass at the site of the right chest wall drainage bag. The tumor gradually increased in size, and was resected in 2012, without pathological examination 


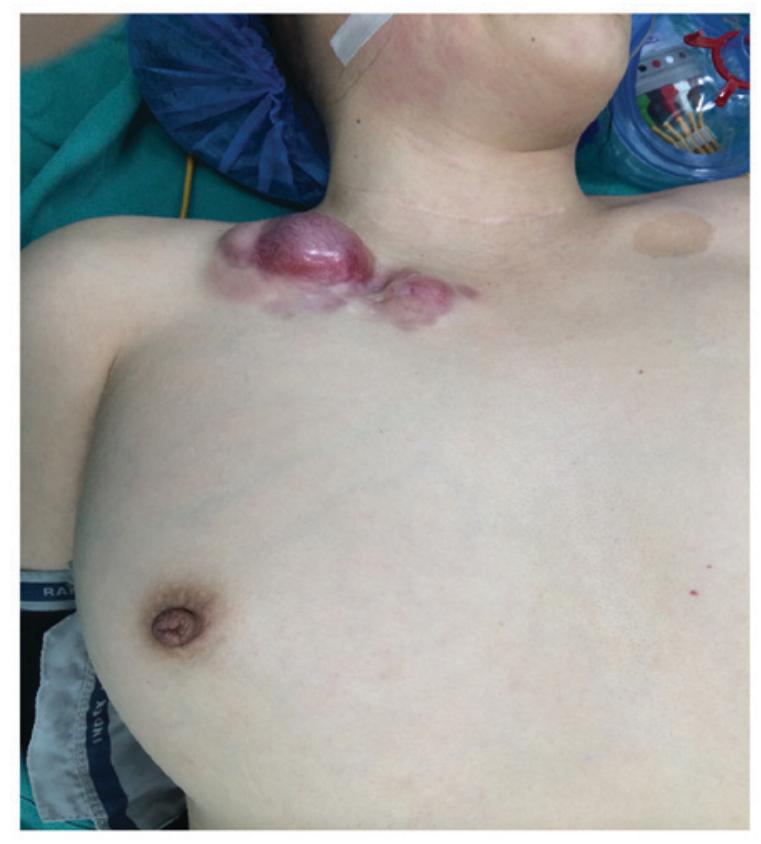

Figure 2. Case 1: Skin lesion of patient.

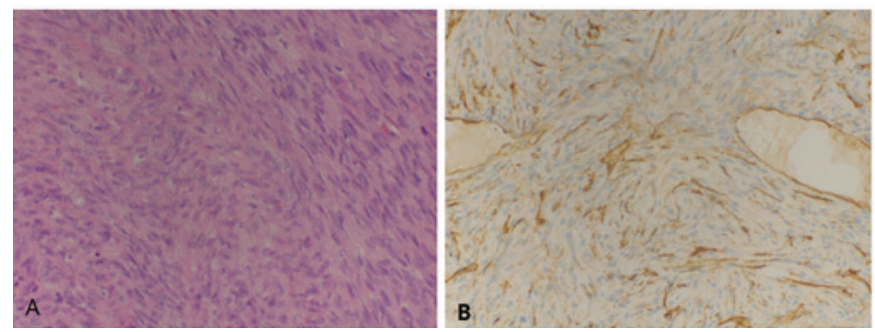

Figure 3. Case 1: (A) Hematoxylin and eosin staining, and (B) immunohistochemistry demonstrating a cluster of differentiation 34(+) result (magnification, $\mathrm{x} 200$ ).

of the right chest wall. A red tumor recurred in the same place in 2014, which was evident on the body surface. The tumor was hard, smooth, irregular in shape, and measured $5.2 \times 2.5 \times 3.0 \mathrm{~cm}$, with no pain to the touch, no bleeding, and no rupture or ulceration. The surrounding skin was reddish in color and uneven, resembling a keloid. Telangiectasia was evident on the surface (Fig. 2). During surgery, the resection was expanded $0.2 \mathrm{~cm}$ from the tumor rim. However, examination of rapid-freeze biopsy showed a low-degree of malignant chest wall spindle cell tumor, therefore, the surgeon expanded the resection margin to $3 \mathrm{~cm}$. The postoperative pathological diagnosis was FS-DFSP of the chest wall. Immunohistochemistry demonstrated CD34(+) (Fig. 3) and Ki-67(20\%+) results. The tumor recurred in 2016, and preoperative positron emission tomography-computed tomography (CT) revealed distant bone metastases (Fig. 4). The patient received no further surgical treatment and succumbed to mortality in 2017.

Case 2. Case 2 was of a 57-year-old woman who had undergone surgical resection for a tumor at the top of her head at a local hospital in 2007, without pathological examination. The tumor recurred twice in the same site in 2013 and 2015, and the patient underwent surgery on both occasions, without pathological

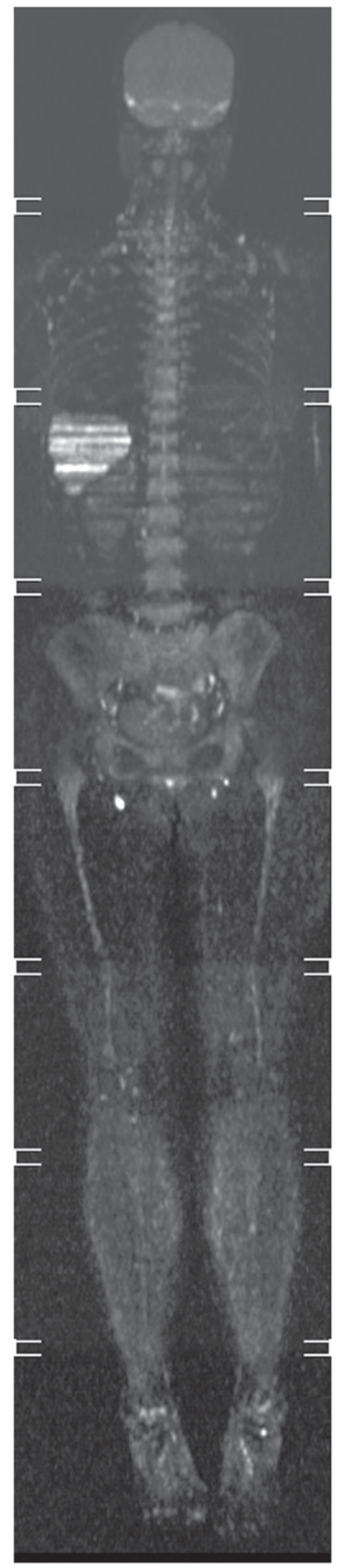

Figure 4. Case 1: Preoperative positron emission tomography-computed tomography revealed distant bone metastases. 


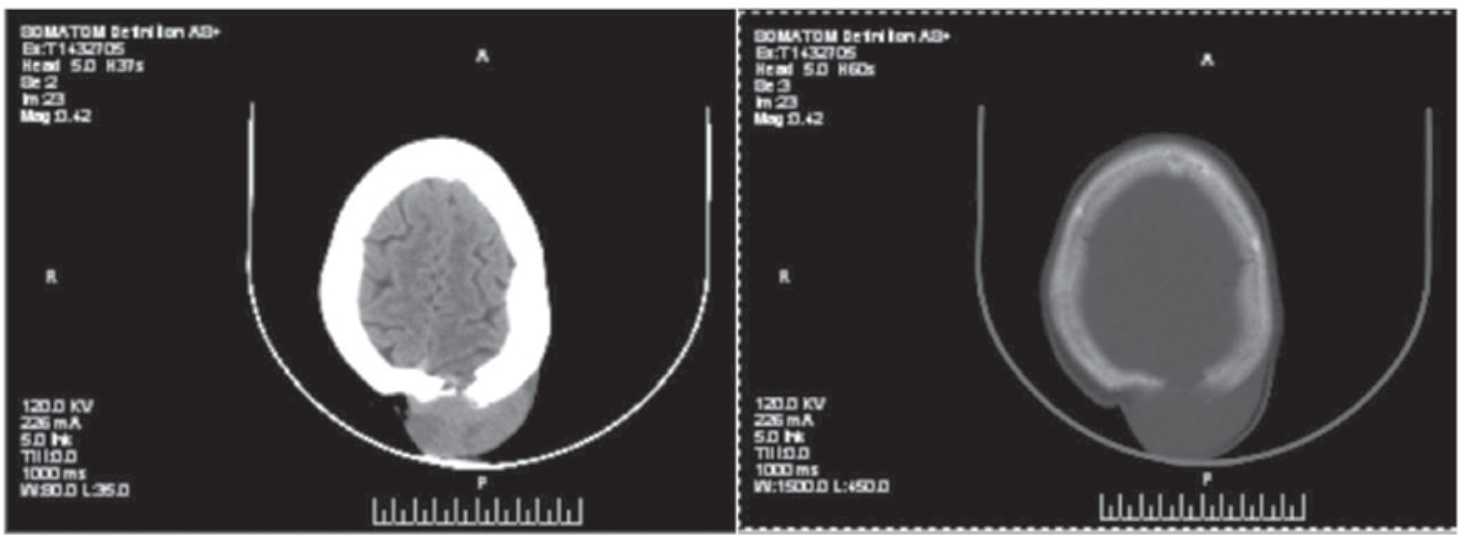

Figure 5. Case 2: Preoperative computed tomography examination revealed a subcutaneous tumor at the left side of the top of the patient's head.
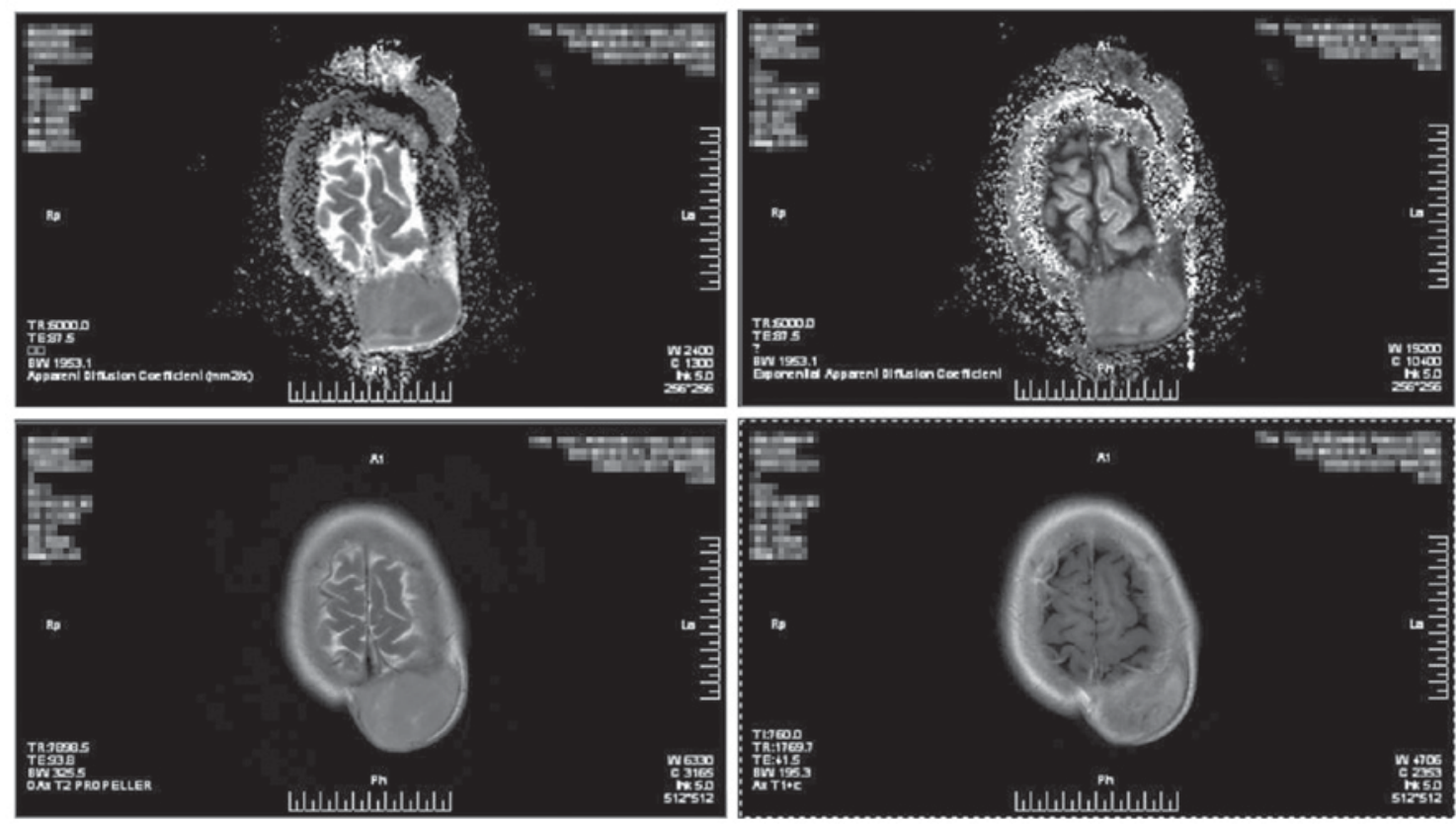

Figure 6. Case 2: Magnetic resonance imaging suggested involvement of the adjacent skull.

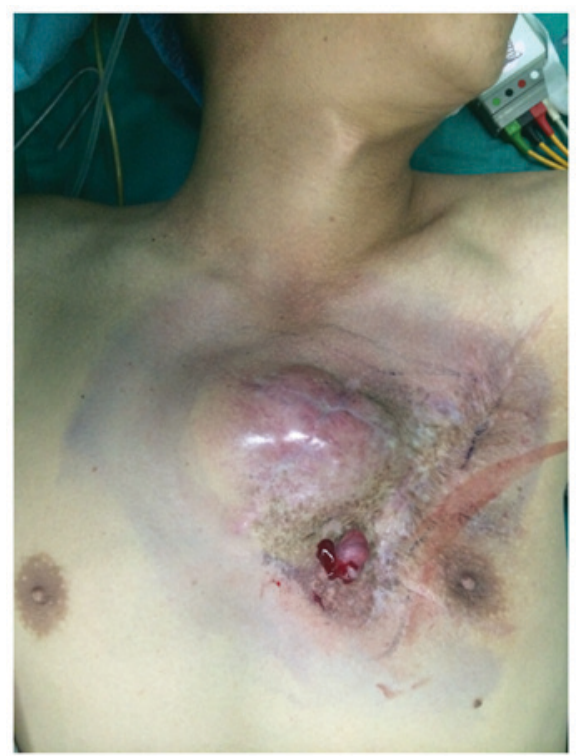

Figure 7. Case 3: Skin lesion with red and cauliflower-like appearance. examination. The patient attended the Department of Plastic Surgery, The First Affiliated Hospital of Zhengzhou University for treatment of the fourth recurrence in 2016. Preoperative CT examination revealed a subcutaneous tumor on the left side of the top of her head, and magnetic resonance imaging suggested involvement of the adjacent skull (Figs. 5 and 6). To avoid bleeding during surgery, internal vascular embolization of the tumor was initially performed, and the resection was then expanded to $3 \mathrm{~cm}$, with confirmation that the margin was negative. The wound was closed using pedicled flaps. Postoperative pathological examination suggested that the tumor was FS-DFSP. Immunohistochemistry revealed CD34(+) and $\mathrm{Ki}-67(15 \%+)$. The postoperative blood supply to the flaps was poor, and flap necrosis was observed 2 weeks following surgery. However, the wound healed, and there was no sign of tumor recurrence for 1.5 years.

Case 3. Case 3 was of a 46-year-old man, who developed a $2.3 \times 2.5 \mathrm{~cm}$ lump in 1976 , following a chest-wall injury. The 

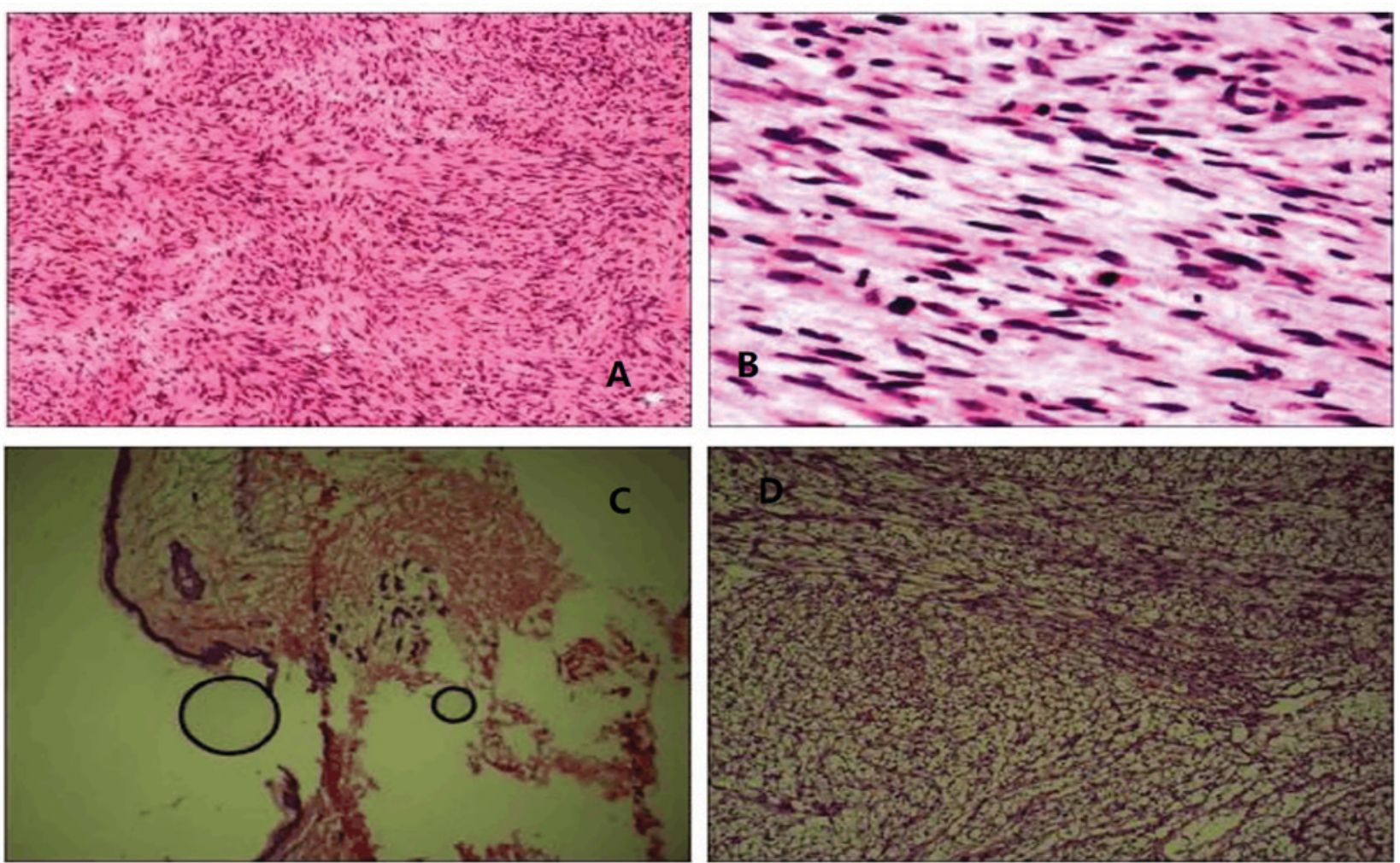

Figure 8. Case 3: (A) magnification, x100 and (B) magnification, x400 images from the patient examination in 2016, with a diagnosis of fibrosarcomatous DFSP. (C) magnification, x40 and (D) magnification, x100 images from the patient examination in 2014, with a diagnosis of DFSP. All four images were stained using hematoxylin and eosin staining. DFSP, dermatofibrosarcoma protuberans.
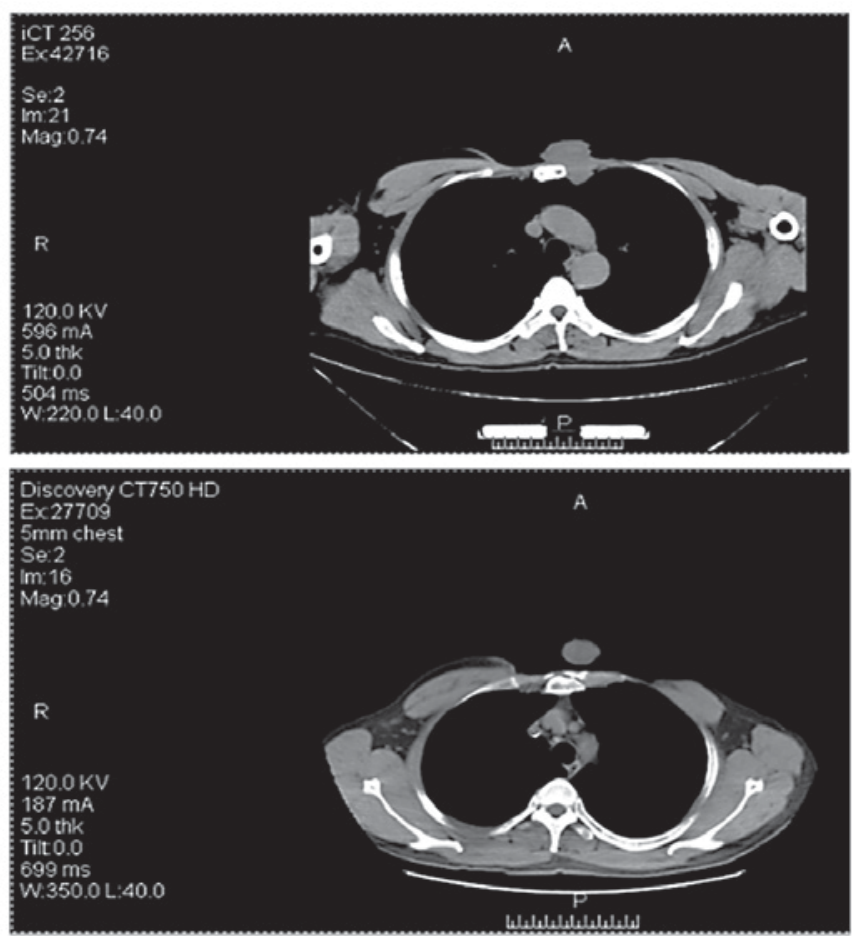

Figure 9. Case 3: Tumor recurrence occurred twice in 2014 and 2015, which invaded the chest wall.

lump was not pruritic or painful, and the patient sought no attention for it. However, the tumor had grown to adult-fist size by 2008 , and the patient underwent chest wall tumor

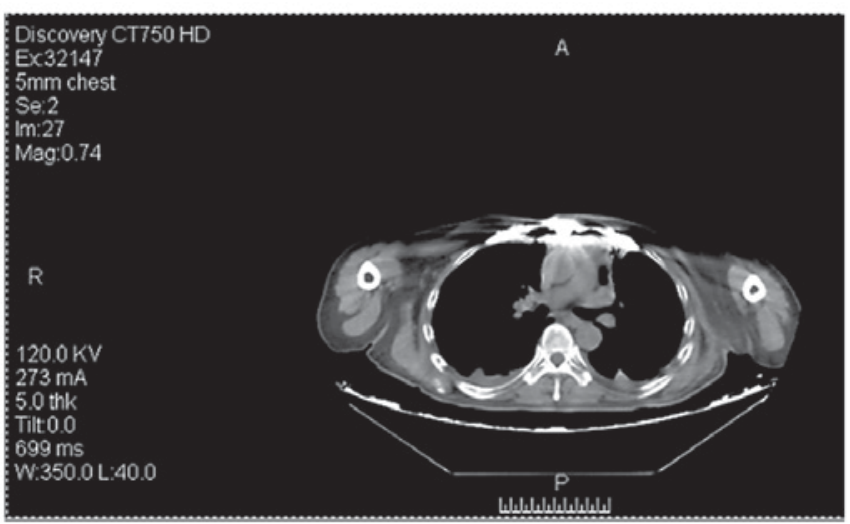

Figure 10. Case 3: Chest computed tomography indicated that the tumor had invaded the ribs, and the majority of ribs on one side were missing.

resection at another hospital. The intraoperative and postoperative pathological diagnoses were of DFSP. In 2014, the tumor reappeared in the original position. It was red and cauliflower-like in appearance, and increased gradually to $10.0 \times 8.0 \mathrm{~cm}$. A red, cauliflower-shaped mass of about $1.0 \times 1.0 \mathrm{~cm}$ was found in the lower right of the tumor. The hard surface was free of rupture and bleeding (Fig. 7). The resection was expanded to $3 \mathrm{~cm}$ and the wound was closed using a skin graft, followed by vacuum sealing negative pressure drainage for 9 days following surgery. The skin graft survived well. The results of intraoperative frozen biopsies showed negative margins. Immunohistochemistry revealed CD34(+) and Ki-67(30\%+) (Fig. 8). However, the tumor recurred twice 
in 2014 and 2015 (Fig. 9), respectively, and invaded the chest wall. An expanded resection was performed without removal of the ribs, however, the tumor recurred in 2016 at the original site. A chest CT indicated that the tumor had invaded the ribs, and the majority of the ribs on one side were missing (Fig. 10). Another resection was performed, and chest surgery was arranged to repair the ribs.

\section{Discussion}

Clinically, DFSP often masquerades as a benign, indolent tumor. Microscopically, it extends far beyond the assessed clinical margins, spreading in the dermis and subcutaneous tissue (3). DFSP has a distinctive histologic appearance but can mimic other diseases. Histologically, several variants of DFSP have been described and it is important they are well characterized to avoid misdiagnosis with other types of tumor. These variants include pigmented, myxoid, myoid, granular cell, sclerotic, atrophic DFSP and giant cell fibroblastoma (2). At this point, it is necessary to distinguish the tumor from other tumors. The high incidence of misdiagnosis of DFSP highlights the importance for pathological examination of early skin lesions in order to clarify the diagnosis $(9,10)$. Histology with immunohistochemistry remain the gold standard for ensuring an accurate diagnosis $(11,12)$. DFSP may be a more aggressive tumor, its behavior can be affected by surgical treatment (13). The standard treatment is wide local resection with at least a 2-cm margin (2). According to the literature, the recurrence rate of DFSP following expansion to $2 \mathrm{~cm}$ remains high, whereas the recurrence rate was significantly reduced following expansion to $>3 \mathrm{~cm}$ (14-17). Therefore, the resection was expanded to $3 \mathrm{~cm}$ in all later cases, and negative margins were confirmed by pathological examination of intraoperative frozen specimens.

The total postoperative recurrence rate in the present study was $25.7 \%$. Based on available retrospective data, the risk of metastasis and recurrence is elevated in FS-DFSP, compared with that in DFSP. In 2015, Hoesly et al (9) also reported that DFSP-FS exhibited more aggressive behavior than DFSP, with lower recurrence-free survival rate and increased metastatic potential. Furthermore, recurrent cases are more likely to relapse following treatment than primary cases. Five patients $(7 \%)$ received postoperative radiotherapy for positive margins following maximal excision. These five patients showed no recurrence at the time of reporting. DuBay et al (1) reported that wide local excision with careful pathologic analysis of margins had a low recurrence rate and was used for the majority of patients with DFSP lesions. Mohs micrographic surgery (MMS) entails a more elaborate technique and is particularly suitable for cases of DFSP where an extended excision is difficult (3). A study by Paradisi et al (18) showed that the recurrence rates of MMS were significantly lower than those of WLE. Although this technique is frequently used in certain countries, it was not available at The First Affiliated Hospital of Zhengzhou University due to technical constraints.

The main limitation of the present study is that was a retrospective analysis, and the method of surgery was not the most advanced. Compared with other studies, the follow-up time was also short and, with time, there may be different results. Therefore, a large prospective randomized trial (stratified random sampling) and multicenter study is necessary to demonstrate possible differences.

In conclusion, DFSP is a rare disease and an improved awareness and understanding of this condition is required by surgeons and pathologists to allow its early diagnosis and treatment. Pathological examinations are required in patients with suspected DFSP, with the aim of minimizing the misdiagnosis rate. Once diagnosed, DFSP requires prompt treatment by extended tumor resection, followed by an increased follow-up frequency. Combined treatment requires consideration to reduce the recurrence rate in unresectable cases or in patients with repeated recurrence following resection. When surgery is insufficient or disease is metastatic, imatinib may be more important in treatment in the future $(7,19,20)$. These data may be useful to assist clinicians when they treat patients with DFSP. In subsequent investigations, combining MMS with radiation or chemotherapy is worth consideration.

\section{Acknowledgements}

The authors would like to thank The First Affiliated Hospital of Zhengzhou University for providing clinical information and Mr. Erwei Xu (Zhengzhou University) for assistance with the photographs.

\section{Funding}

No funding was received.

\section{Availability of data and materials}

All data generated or analyzed during this study are included in this published article.

\section{Authors' contributions}

QW was responsible for the conception and design of the present study and revised the manuscript. AL performed data analysis and drafted the manuscript. Both authors interpreted results, and read and approved the final version of the paper. The authors confirm that the content has not been published elsewhere and does not overlap their published work.

\section{Ethics approval and consent to participate}

The study was approved by the Ethics Committee of The First Affiliated Hospital of Zhengzhou University. Information was collected from the medical records of patients, with no influence on diagnosis or treatment. Prior to the study, patients were fully informed to clarify the purpose and significance of the study.

\section{Consent for publication}

The patient or guardian provided written informed consent for the publication of any associated data and accompanying images. 


\section{Competing interests}

The authors declare that they have no competing interests.

\section{References}

1. DuBay D, Cimmino V, Lowe L, Johnson TM and Sondak VK: Low recurrence rate after surgery for dermatofibrosarcoma protuberans: A multidisciplinary approach from a single institution. Cancer 100: 1008-1016, 2004.

2. Llombart B, Serra-Guillén C, Monteagudo C, López Guerrero JA and Sanmartin O: Dermatofibrosarcoma protuberans: A comprehensive review and update on diagnosis and management. Semin Diagn Patho 30: 13-28, 2013.

3. Snow SN, Gordon EM, Larson PO, Bagheri MM, Bentz ML and Sable DB: Dermatofibrosarcoma protuberans: A report on 29 patients treated by Mohs micrographic surgery with long-term follow-up and review of the literature. Cancer 101: 28-38, 2004.

4. Hoffman E: Über das knollentreibende fibrosarkom der haut (Dermatofibrosarkoma protuberans). Dermat Ztschr 43: 1-28, 1925. (In German)

5. Al-Rahbi S, Al-Lawati T, Al-Kharusi S, Thomas S and Al-Harrasi K: Dermatofibrosarcoma Protuberans: A rare malignancy of the breast. Oman Med J 30: 378-381, 2015.

6. Bogucki B, Neuhaus I and Hurst EA: Dermatofibrosarcoma protuberans: A review of the literature. Dermatol Surg 38: 537-551, 2012

7. Fletcher CD: The evolving classification of soft tissue tumours-an update based on the new 2013 WHO classification. Histopathology 64: 2-11, 2014.

8. Sigel JE, Bergfeld WF and Goldblum JR: A morphologic study of dermatofibrosarcoma protuberans: Expansion of a histologic profile. J Cutan Pathol 27: 159-163, 2000.

9. Hoesly PM, Lowe GC, Lohse CM, Brewer JD and Lehman JS Prognostic impact of fibrosarcomatous transformation in dermatofibrosarcoma protuberans: A cohort study. J Am Acad Dermatol 72: 419-425, 2015.

10. Iorizzo LJ III and Brown MD: Atypical fibroxanthoma: A review of the literature. Dermatol Surg 37: 146-157, 2011.
11. Karanian M, Perot G, Coindre JM, Chibon F, Pedeutour F and Neuville A: Fluorescence in situ hybridization analysis is a helpful test for the diagnosis of dermatofibrosarcoma protuberans. Mod Pathol 28: 230-237, 2015.

12. Saiag P, Grob JJ, Lebbe C, Malvehy J, del Marmol V, Pehamberger $\mathrm{H}$, Peris $\mathrm{K}$, Stratigos A, Middelton $\mathrm{M}$, Basholt L, et al: Diagnosis and treatment of dermatofibrosarcoma protuberans. European consensus-based interdisciplinary guideline. Eur J Cancer 51: 2604-2608, 2015.

13. Szollosi Z and Nemes Z: Transformed dermatofibrosarcoma protuberans: A clinicopathological study of eight cases. J Clin Pathol 58: 751-756, 2005.

14. Popov P, Bohling T, Asko-Seljavaara S and Tukiainen E: Microscopic margins and results of surgery for dermatofibrosarcoma protuberans. Plast Reconstr Surg 119: 1779-1784, 2007.

15. Tom WD, Hybarger CP and Rasgon BM: Dermatofibrosarcoma protuberans of the head and neck: Treatment with Mohs surgery using inverted horizontal paraffin sections. Laryngoscope 113: 1289-1293, 2003.

16. Pallure V, Dupin N and Guillot B; Association for Recommendations in Dermatology: Surgical treatment of Darier-Ferrand dermatofibrosarcoma: A systematic review. Dermatol Surg 39: 1417-1433, 2013.

17. Kurlander DE, Martires KJ, Chen Y, Barnholtz-Sloan JS and Bordeaux JS: Risk of subsequent primary malignancies after dermatofibrosarcoma protuberans diagnosis: A national study. J Am Acad Dermatol 68: 790-796, 2013.

18. Paradisi A, Abeni D, Rusciani A, Cigna E, Wolter M, Scuderi N, Rusciani L, Kaufmann R and Podda M: Dermatofibrosarcoma protuberans: Wide local excision vs. Mohs micrographic surgery. Cancer Treat Rev 34: 728-736, 2008

19. Mendenhall WM, Zlotecki RA and Scarborough MT: Dermatofibrosarcoma protuberans. Cancer 101: 2503-2508, 2004.

20. Tazzari M, Indio V, Vergani B, De Cecco L, Rini F, Negri T, Camisaschi C, Fiore M, Stacchiotti S, Dagrada GP, et al: Adaptive immunity in fibrosarcomatous dermatofibrosarcoma protuberans and response to imatinib treatment. J Invest Dermatol 137: 484-493, 2017. 